\title{
Wide QRS Tachycardias: the Rationale Behind Electrocardiographic Diagnostic Criteria
}

Leonardo Filipe Benedeti Marinucci ${ }^{1, *}$, William Azem Chalela ${ }^{1}$

\section{ORCID IDs}

Marinucci LFB (D) https://orcid.org/0000-0002-0821-0219

Chalela WA (D) https://orcid.org/0000-0003-3751-0728

\begin{abstract}
Wide QRS tachycardias represent one of the most challenging scenarios in the interpretation of the electrocardiogram, even among experienced professionals or specialists. The various existing diagnostic criteria are essential tools for the correct identification of the origin of tachycardia, however, the knowledge of the electrophysiological principles of cardiac depolarization is fundamental to understand them, thus allowing greater accuracy in the interpretation of the exam.
\end{abstract}

KEYWORDS: Tachycardia; Diagnosis; Criteria

1. Universidade de São Paulo-- Faculdade de Medicina -Instituto do Coração do Hospital das Clínicas - São Paulo (SP), Brazil. *Correspondonding author: leofilipe96@gmail.com

Received: Jun 18, 2020 | Accepted: Jun 22, 2020 


\section{INTRODUCTION}

Tachycardias that present with a wide $\mathrm{QRS}$ on the electrocardiogram (duration $\mathrm{QRS} \geq 120 \mathrm{~ms}$ ) are usually a cause for diagnostic uncertainty and can generate anxiety and insecurity in the conduct of the clinical case, since the differentiation between supraventricular or ventricular origin is not always simple and intuitive, despite being fundamental for the proper management of the patient, with etiologies, treatment and prognosis very different from each other. If we add to that the emergency character normally related to these cases, when decisions must be quick and there is less scope for errors, and the negative impact that incorrect diagnosis can have on clinical outcomes ${ }^{1}$, we are facing a scenario that is most challenging for the doctor, and that puts his confidence to the test.An example of this is the high percentage of disagreement in the diagnosis of wide QRS tachycardias among observers in the emergency room, reaching values of $50 \%$ in some studies, and a diagnostic accuracy only moderate in that same context, around $70 \%{ }^{2}$, even using established algorithms that have proven to improve these variables. Even when more experienced professionals with specialized training were tested, the specificity values ranged from 43 to $70 \%^{3}$, still far from ideal.

Thus, understanding the physiological mechanisms of cardiac activation that explain the most used criteria for differential diagnosis of wide QRS tachycardias, and thus being able to deduce them instead of just memorizing them, appears as a way to improve the electrocardiographic interpretation in doubtful cases.

\section{CLINICAL PRESENTATION AND PAST PATHOLOGICAL HISTORY}

Anamnesis and physical examination data have been the pillars of clinical reasoning and the development of diagnostic hypotheses since the beginning of medicine, and remain fundamental even in the era of technological evolution, where complementary examinations have assumed a leading role in medical evaluation. In the case of wide QRS tachycardias, there is no history data or alterations in physical examination that allow the diagnosis dilemma to be elucidated, nor should they be the decisive factor in the elaboration of the electrocardiographic report, but auxiliary tools in the composition of the complete reasoning and that, associated with changes in the electrocardiogram (ECG), will define the most appropriate conduct.

It is known that the most common cause of wide QRS tachycardia on the ECG is ventricular tachycardia, in the order of $80 \%$ of cases, followed by supraventricular tachycardias with previous branch aberration or block (15\%) and "pre-excited (5\%), that is, conducted in an anterograde way by an accessory path ${ }^{4}$. If we consider a population like that of patients with a history of myocardial infarction or heart failure, the positive predictive value for VT reaches values above $95 \% 5$.

In short, simple anamnesis is able to establish the pre-test probability which, associated with the prevalence of structural heart disease, can predict a high chance of tachycardia being of ventricular origin. Based on the above, and considering that the effect of medications used to treat supraventricular tachycardias, such as beta-blockers and calcium channel blockers, can lead to hemodynamic deterioration and adverse clinical outcomes in VT, it is reasonable to admit the diagnosis of ventricular tachycardia until proven otherwise ${ }^{6}$. In a scenario where uncertainty predominates, the criteria set out below are of great value to increase the accuracy of electrocardiographic interpretation.

\section{ELECTROCARDIOGRAPHIC ASPECTS IN THE DIFFERENTIAL DIAGNOSIS OF WIDE QRS TACHYCARDIAS}

\section{Atrioventricular dissociation}

Atrioventricular dissociation (AV) - lack of relationship between depolarization of the atria and ventricles, in that order - is an indirect sign of the ventricular origin of tachycardia and one of the most remembered among the diagnostic criteria in wide QRS tachycardias, due to the easy understanding of its physiological mechanism. Ventricular contraction at a higher frequency and with no chronological relationship to atrial contraction suggests that the stimulus for depolarization of the 
ventricles is located below the AV node and does not depend on the one that controls the depolarization of the atria. In addition, there are few and rare conditions that also occur with this finding, such as unusual forms of Mahaim tachycardia (accessory pathways that communicate the atria to the infra-hisian conduction system). This makes it a great predictor of ventricular tachycardia (VT) when found, giving it a specificity close to $100 \%$, as observed in the study of Brugada that listed it as one of the diagnostic criteria in its algorithm ${ }^{7}$.

Despite this, the low sensitivity (values such as $21 \%^{7}$ ) appears as a limitation in its applicability in isolation. This is due to the difficulty in identifying the $\mathrm{P}$ wave amidst the tachycardic rhythm, often hidden within the QRS or $\mathrm{T}$ wave, and to the ventriculo-atrial conduction present in up to $50 \%$ of the $\mathrm{VT}^{4}$, mimicking a $1: 1$ conduction ratio between the two chambers (Fig. 1). There is a greater chance of detecting AV dissociation in leads where the $\mathrm{P}$ wave is more prominent (lower leads and V1), and the use of the Lewis lead is recommended ${ }^{8}$ (obtained in lead D1, by placing the electrodes of the right and left arms adjacent to the right sternal border, in the second and fourth intercostal spaces, respectively), to make their visualization easier and increase their sensitivity.

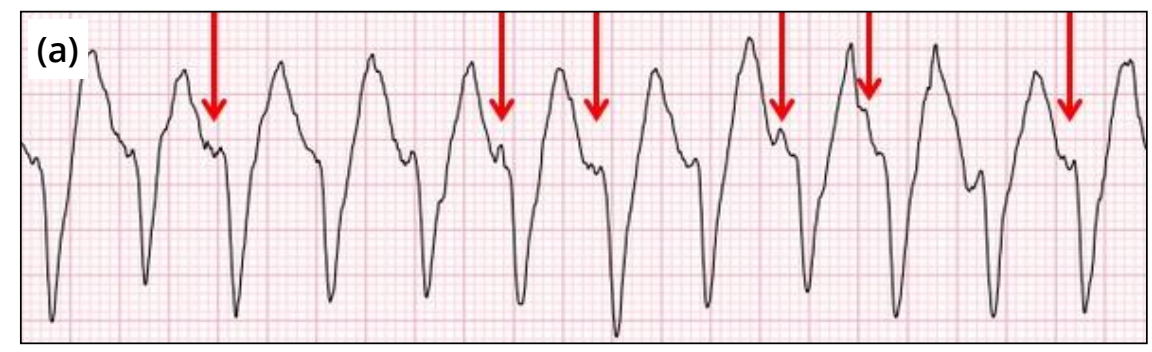

(b)

Key points: AV dissociation

P waves that do not conduct QRS complexes

Contains high probability of VT

- Present in only $20 \%$ of VTs

Figure 1. (a) AV dissociation: $P$ waves are observed with no conduction relationship with the QRS complexes. (b) Main topics related to $A V$ dissociation.

\section{QRS morphological analysis}

When AV dissociation is not present or cannot be easily identified, the QRS complex morphology must be evaluated in different leads to, based on the characteristics of the vectors that form it (polarity, amplitude and duration), presume the origin of the tachycardia. This may be a limiting step for many professionals, as the lack of knowledge about the basic electrophysiological mechanisms of cardiac activation prevents the understanding of the foundations of the changes found, requiring simple memorization of the criteria; this favors quick forgetfulness with disuse and difficulty in interpreting atypical presentations. Therefore, by understanding how the normal QRS complex is formed, it is easier to explain the mechanism of the changes seen in pathological situations, and thus to diagnose them.

\section{Normal ventricular activation sequence}

The electrocardiogram is nothing more than the cartographic representation of cardiac electrical activation, that is, it is the projection of a three-dimensional image, drawn by the progression of the electrical impulse along the different parts of the heart, in two two-dimensional planes perpendicular to each other (frontal and horizontal). It is like "drawing the path" that the electrical stimulus travels from different angles: seen from above (which shows whether it is moving forward or backward) or seen from the front (which shows whether it goes up or down), in addition to movement right or left. In normal activation, the impulse that comes from the atria through the conduction system initially depolarizes the interventricular septum, through which the His-Purkinje fibers run, initially on its left side, generating a vector oriented to the right and forward, inscribing q waves initials in the negativity of leads D1 and $\mathrm{V} 6$, and $\mathrm{r}$ waves in the positivity of V1 and V2. Then, the free walls of the right and left ventricles are activated, originating a resulting vector facing left, down and back, due to the predominance of the left ventricular (LV) muscle mass, inscribing $\mathrm{S}$ waves in the negativity of V1 and V2 and R wave in V5 and V6 positivity. Finally, the basal areas 
of the ventricles are activated, places with less nerve endings, generating a vector oriented to the right, up and back, inscribing s waves in the V6 negativity ${ }^{9}$ (Fig. 2A).

\section{Sequence of ventricular activation in right and left bundle branch blocks}

Even in the presence of a branch block, the initial septal activation is preserved; in the right bundle branch block (RBBB) it follows the normal pattern, with the vector facing right, due to the left dominance already observed, and in the left bundle branch block (LBBBB) there is a vector inversion, being now oriented to the left by activation initial septum and right ventricle, explaining the absence of $\mathrm{Q}$ wave in V6. In RBBB, LV activation completes an initial portion of the QRS that is very similar to normal, with a final portion altered at the expense of a vector turned to the right, corresponding to the slow and abnormal activation of the RV, inscribing an enlarged $\mathrm{R}$ 'wave in $\mathrm{V} 1$ and $\mathrm{S}$ wave in V6(Fig. 2b). In LBBB, the slow and abnormal activation of the LV generates a second vector with a similar orientation to the normal, but with a prolonged duration, generating enlarged and notched complexes in the precordial leads, and positive in leads V5, V6 and D1 (type R), by masking of the other vectors that depolarize other regions simultaneously with the delayed activation of the LV (Fig. 2c).
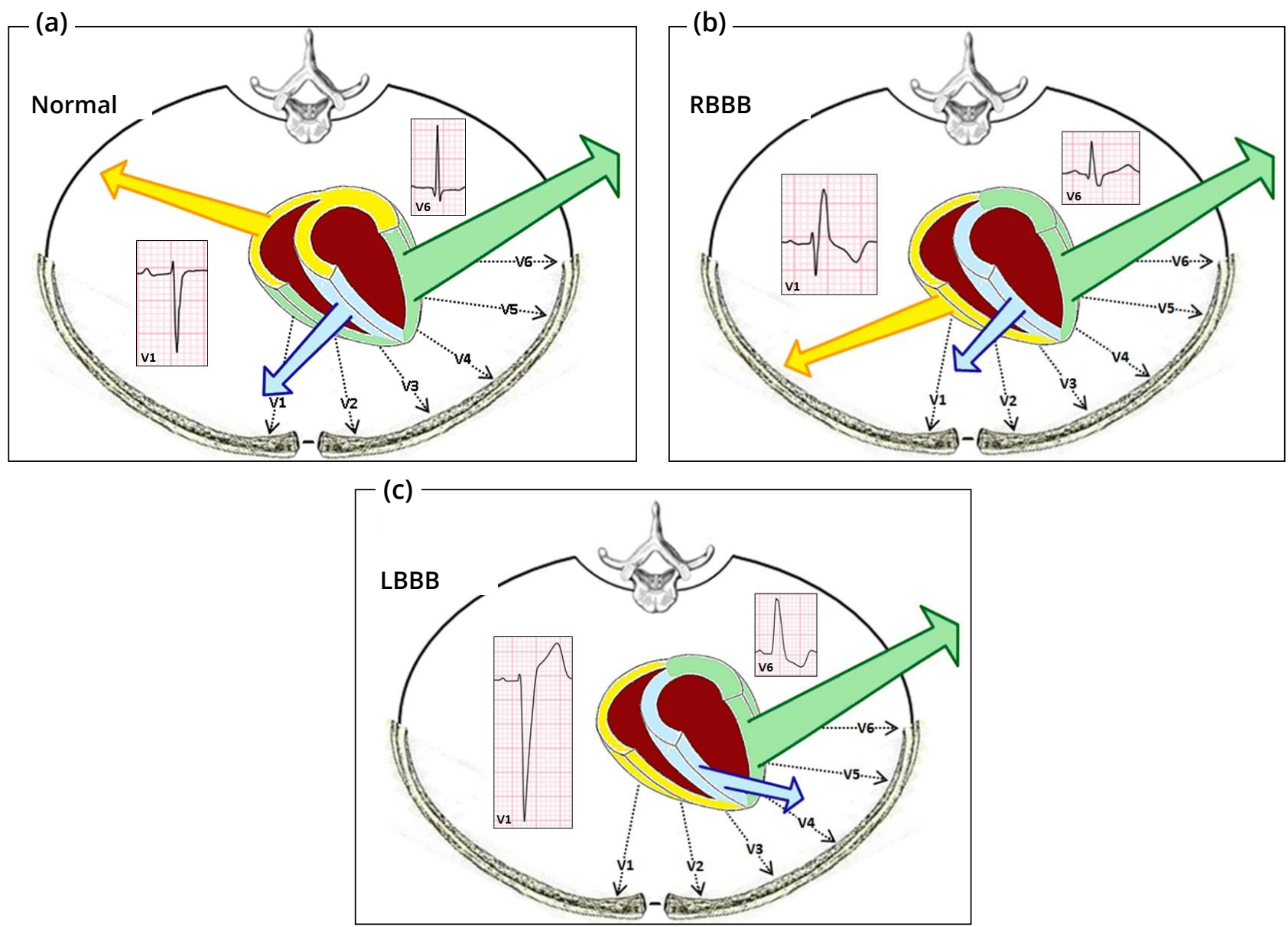

Figure 2. Cardiac activation vectors, in normal depolarization (a) and in the right (b) and left (c) branch blocks.

\section{Morphological aspects that suggest the origin of tachycardia}

Based on the morphological aspects of the QRS complex described above, we observed some characteristics present both during normal AV conduction and in the presence of bundle branch blocks. Thus, it is possible to assume the ventricular origin of tachycardia, when the anomalous activation of cardiac structures, from an autonomous ventricular focus, generates a morphological pattern with its own characteristics, due to the change in the resulting depolarization vectors. 


\section{Polarity in precordials and aVR}

In supraventricular tachycardias, which depolarize the ventricles through the conduction system, the initial activation of the septum, followed by the activation of the ventricles, generates vectors in different directions, and sometimes opposite to each other in some derivations of the electrocardiogram. Thus, it is unlikely that a supraventricular tachycardia with aberrant conduction will produce strictly positive or negative QRS complexes in all precordial leads (without waves with opposite polarities in relation to the baseline, that is, the absence of complexes with RS morphology), making this very specific pattern of ventricular tachycardias (100\% specificity in the study of Brugada that places it as one of the diagnostic criteria in his algorithm), despite low sensitivity $(26 \%)^{7}$.

In similar reasoning, the positive or negative agreement between QRS complexes in the precordial leads (all complexes facing up or down in relation to the baseline) has high specificity for $\mathrm{VT}^{10}$, despite the low sensitivity of this criterion (present in only about $20 \%$ of $\mathrm{VT}^{6}$ ). If we also analyze the polarity of the $\mathrm{QRS}$ complex in the aVR lead, located in the frontal plane, we will notice that the supraventricular stimulus that depolarizes the ventricles through the conduction system, in the topdown direction, moves away from this lead and generates a vector opposite to it, entering a negative initial wave in aVR (Qwave) (Fig. 3). This makes the presence of a positive initial wave in this lead ( $\mathrm{R}$ wave) a finding with high specificity for VT, close to $99 \%$, as observed in the study by Vereckei that places it as one of the diagnostic criteria in his algorithm ${ }^{11}$.

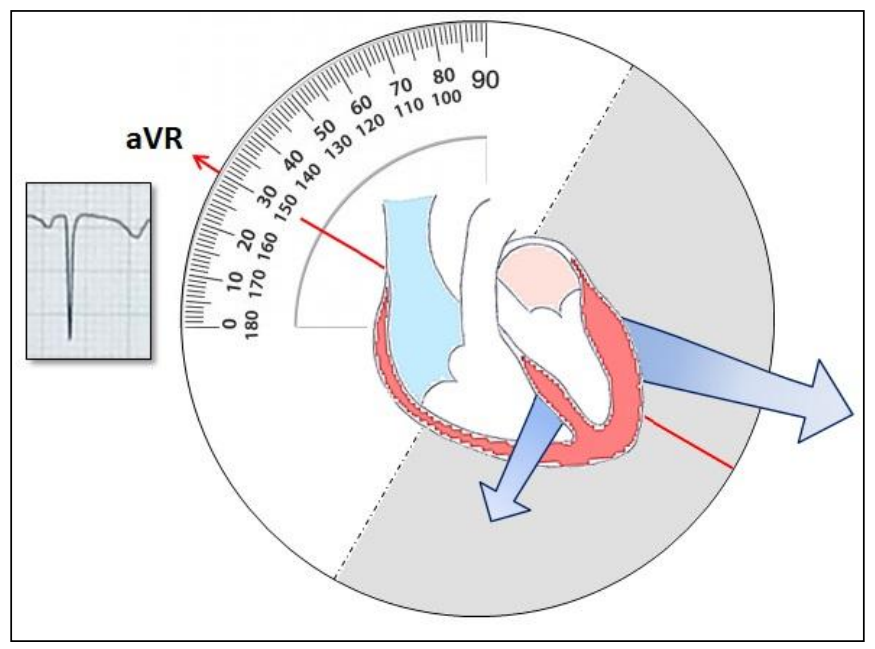

Figure 3. Normal depolarization of the septum / left ventricle generates vectors that oppose the aVR lead in the frontal plane, inscribing a negative initial wave in this lead.

\section{Morphology in V1 and V6}

In supraventricular tachycardias with conduction aberration, it is expected to find a morphological pattern with typical features of branch block, in antagonistic leads V1 and V6. When an anomalous branch block pattern is observed, which differs from the electrophysiological characteristics expected in this condition, it becomes very suggestive of being of ventricular origin.

In the case of a VT with RBBB morphology, a wavefront progresses from the left ventricle towards the right precordial lead $\mathrm{V} 1$, forming a prominent $\mathrm{R}$ wave pattern in that lead (pure $\mathrm{R}, \mathrm{Rsr}$ 'or $\mathrm{qR}$ ), as opposed to the three-phase pattern seen in the classic $\mathrm{RBBB}$, with an initial $\mathrm{r}$ wave corresponding to the activation of the septum, an $\mathrm{S}$ wave that departs from V1 (LV activation) and $\mathrm{R}$ 'corresponding to the slow activation of the RV. In V6 the RS pattern corresponds to the initial activation of the LV (R wave) accompanied by the slow activation of the RV ( $\mathrm{S}$ wave), which causes the voltage of the $\mathrm{R}$ wave to be higher than that of the $\mathrm{S}$ wave, due to the difference in mass between the ventricles $(R>S)$. When an $R<S$ pattern is observed, the ventricular origin becomes suspect.

In the case of a VT with LBBB morphology, there is a wavefront that progresses from right to left, moving away from V1 as in SVT with left bundle branch block; however, in VT, the stimulus is conducted through the myocardium, while in SVT it initially travels through the intact right branch. Thus, despite having the same vectorial orientation (from right to left, moving away from V1), their morphological characteristics are different; the impulse of ventricular origin presents an enlarged initial $\mathrm{R}$ wave (> 30 
$\mathrm{ms}$ ), as well as the duration of the $\mathrm{S}$ wave ( $>60 \mathrm{~ms}$ ), which may present notches in its descending phase due to the greater difficulty in conducting the stimulus through the muscle tissue. In V6, the presence of Q wave is very suggestive of VT, indicating that the wavefront moves away from the apical region of the $L V$ and, therefore, from the left lateral leads, because in LBBB the abnormal initial activation of the septum, from right to right. left, generates a vector oriented in the direction of V6, approaching these leads ${ }^{6,7}$.

\section{QRS complex duration and ventricular activation time}

The speed of the cardiac electrical impulse varies according to the conduction properties of the tissue involved, being faster in the specialized conduction system, and slower through myocardial cells ${ }^{12}$. While a supraventricular stimulus travels through the His-Purkinje system in the process of cardiac activation, an impulse of ventricular origin depolarizes the myocardium through the cardiac muscle cell by cell. The result of this is the widening of the QRS complex not only in its total duration (with values greater than $140 \mathrm{~ms}$ in the RBBB and greater than $160 \mathrm{~ms}$ in the LBBB, suggesting a ventricular origin ${ }^{13}$ ), but mainly at the expense of its initial portion, the so-called "ventricular activation". This phenomenon explains the widening of the initial waves that form the QRS complex, being used as a diagnostic criterion for VT in several algorithms, such as Brugada $^{7}$ (time of the longest interval measured from the beginning of the $\mathrm{R}$ wave to the nadir of the $\mathrm{S}$ wave greater than $100 \mathrm{~ms}$ ), Vereckei ${ }^{11}$ (initial wave $\mathrm{r}$ or q, in the aVR lead, lasting more than $40 \mathrm{~ms}$ ) and Pava ${ }^{14}$ (duration of the interval from the beginning of the QRS to the peak of the $\mathrm{R}$ wave, in lead D2, greater than $50 \mathrm{~ms}$ ), all with high accuracy (greater than $90 \%$ ).

In analogous reasoning, Vereckei establishes a fourth criteria in his algorithm11 which compares the wave amplitude in the first $40 \mathrm{~ms}$ of the QRS complex with the measured amplitude in the final $40 \mathrm{~ms}$ : if the ratio is less than 1, this suggests that the ventricular activation is slower in its initial portion, when it propagates through the muscle, in relation to its final portion, when it can reach parts of the conduction system, increasing its efficiency (Fig. 4). In addition, the presence of notches in the QRS may be related to abnormalities and slowness in intracardiac conduction ${ }^{15}$, and appear as a suggestive criterion of ventricular origin of tachycardia in different studies, such as that by Vereckei (presence of a notch in the downward wave of the initial negative activation) $)^{11}$ and Brugada ${ }^{7}$.

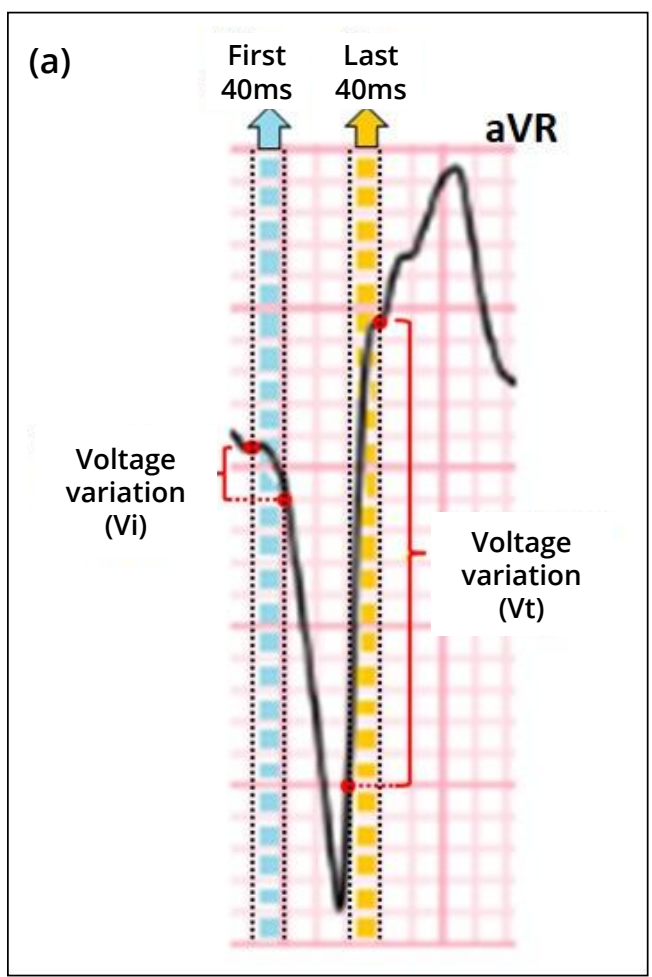

(b)

Key points: QRS morphology

- Bundle branch block with atypical pattern

- Abnormalities of septal waves in precordial waves - Initial positivity in aVR - Increased ventricular activation time and notches on the QRS

Figure 4. (a) Relationship between the variation in amplitude in the initial and final $40 \mathrm{~ms}$ of the QRS complex; when Vi / Vt <1, the rhythm is probably of ventricular origin. (b) Main topics related to QRS morphological analysis in differentiating wide QRS tachycardias. 


\section{Exceptional situations - limitations of morphological criteria}

Despite the high specificity values of the various criteria mentioned, there are situations in which a tachycardia of supraventricular origin may present with electrocardiographic characteristics that suggest a ventricular origin, and vice versa. The morphological criteria in leads V1 and V6 present in the Brugada algorithm, for example, are not met in 4\% of supraventricular tachycardias and in $6 \%$ of ventricular tachycardias, in neither of the two leads; and in up to a third of cases, when the morphology in one derivation suggests a diagnosis, the morphological characteristics in the other favors the opposite $^{7,16}$. Despite the difficulty in differential diagnosis in these cases, the anamnesis data associated with the combination of different electrocardiographic criteria can decrease error rates and increase accuracy; however, situations in which only the invasive study is able to provide this answer are not uncommon.

\section{Ventricular tachycardias with a morphological aspect suggestive of supraventricular origin}

Ventricular tachycardias with a reentry mechanism involving the main branches of the His-Purkinje system (the so-called branch-to-branch VT) or any of the left branch fascicles (the so-called fascicular VT), for using the conduction system in their circuit, depolarizing the myocardium in a relatively concentric manner, they have electrocardiographic characteristics that can resemble a typical branch block, with a narrow QRS when compared to VT originating from other sites, and rapid initial activation ${ }^{17}$. The higher prevalence of fascicular VT in young individuals with no history of structural heart disease may make it even more difficult to suspect a ventricular origin in these cases; the relatively slower heart rate, the morphological aspect of RBBB associated with anterosuperior divisional block of the left branch (ASDBB in 90\% cases) and the therapeutic response to Verapamil may suggest this diagnostic hypothesis. The association of branch-to-branch VT with dilated cardiomyopathy and the previous involvement of the conduction system, observed on the sinus rhythm ECG, may increase the suspicion for the ventricular origin of tachycardia in patients with this morbidity profile ${ }^{18,19}$.

\section{Supraventricular tachycardias with morphological aspect suggestive of ventricular origin}

Tachycardias due to the atrioventricular reentry mechanism that use the accessory pathway (an anomalous muscle bundle that directly connects atria and ventricles) in the anterograde direction, that is, through which the stimulus propagates towards the ventricles, returning to the atria through the conduction, present as tachycardias with wide QRS on ECG. This form of presentation is unusual, being documented in around $5 \%$ of patients with Wolff-Parkinson-White syndrome ${ }^{20}$, and it represents not only a challenge in the differential electrocardiographic diagnosis with ventricular tachycardia, but also a risk condition for these patients. The $\mathrm{QRS}$ origin is at the insertion point of the accessory pathway in the ventricular myocardium, generating a relatively eccentric depolarization sequence with electrophysiological characteristics similar to the ventricular beat, as well as the resulting QRS morphology, which makes the differentiation between both very difficult. Despite the limitation of available criteria, the algorithm by Steurer et al. proposes to assume the origin of the tachycardia, with a sensitivity of $75 \%$ and specificity of $100 \%{ }^{21}$ (Fig. 5).

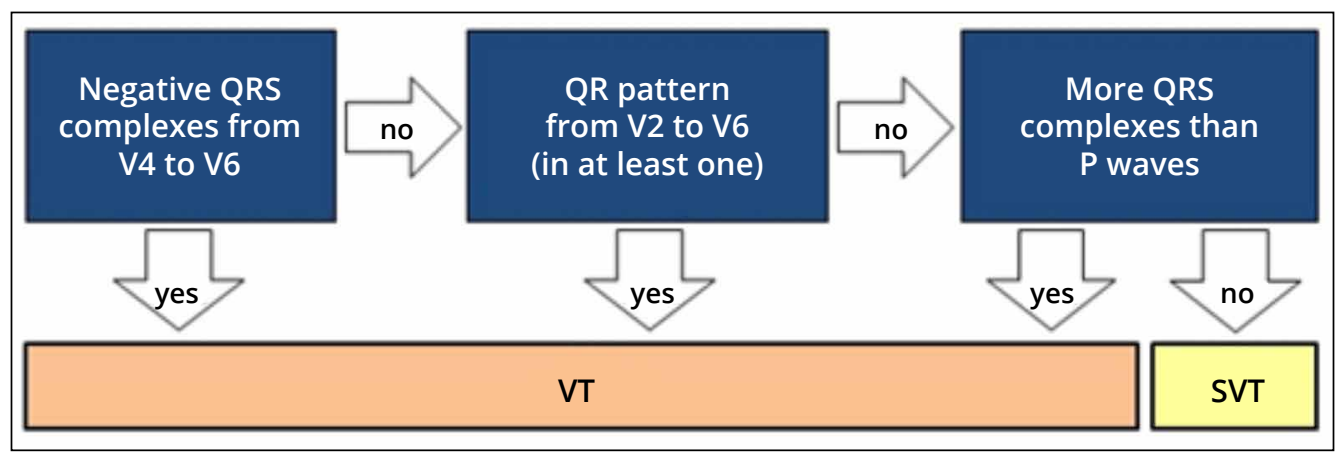

Figure 5. VT algorithm vs. Pre-excited SVT, adapted from Steurer et al ${ }^{21}$. 


\section{CONCLUSION}

The knowledge of the electrophysiological phenomena of cardiac activation allows a better understanding of the diagnostic criteria of wide QRS tachycardias, providing another perspective in the interpretation of the electrocardiogram and making this scenario less challenging in daily medical practice.

\section{REFERENCES}

1. Stewart RB, Bardy GH, Greene HL. Wide complex tachycardia: misdiagnosis and outcome after emergent therapy. Ann Intern Med. 1986;104(6):766-71. https://doi.org/10.7326/0003-4819-104-6-766

2. Baxi RP, Hart KW, Vereckei A, Miller J, Chung S, Chang W, et al. Vereckei criteria used as a diagnostic tool by emergency medicine residents to distinguish between ventricular tachycardia and supra-ventricular tachycardia with aberrancy.J Cardiol. 2012;59(3):30712. https://doi.org/10.1016/j.jjcc.2011.11.007

3. Isenhour JL, Craig S, Gibbs M, Littmann L, Rose G, Risch R. Wide-complex tachycardia: continued evaluation of diagnostic criteria. Acad Emerg Med. 2000;7(7):769-73. https://doi.org/10.1111/j.1553-2712.2000.tb02267.x.

4. Alzand BSN, Crijns HJGM. Diagnostic criteria of broad QRS complex tachycardia: decades of evolution. Europace. 2011;13(4):465-72. https://doi.org/10.1093/europace/euq430

5. Baerman JM, Morady F, DiCarlo LA Jr, de Buitleir M. Differentiation of ventricular tachycardia from supraventricular tachycardia with aberration: value of the clinical history. Ann Emerg Med. 1987;16(1):40-3. https://doi.org/10.1016/S0196-0644(87)80283-4

6. Brugada J, Katritsis DG, Arbelo E, Arribas F, Bax JJ, Blomström-Lundqvist C, et al. 2019 ESC Guidelines for the management of patients with supraventricular tachycardia. The Task Force for the management of patients with supraventricular tachycardia of the European Society of Cardiology (ESC): Developed in collaboration with the Association for European Paediatric and congenital Cardiology (AEPC). European Heart Journal. 2020;41(5):655-720. https://doi.org/10.1093/eurheartj/ehz467

7. Brugada P, Brugada J, Mont L, Smeets J, Andries EW. A new approach to the differential diagnosis of a regular tachycardia with a wide QRS complex. Circulation. 1991;83(5):1649-59. https://doi.org/10.1161/01.cir.83.5.1649

8. Bakker ALM, NijkerkG, Groenemeijer BE, Waalewijn RA, Koomen EM, Braam RL, et al. The Lewis lead: making recognition of P waves easy during wide QRS complex tachycardia. Circulation. 2009;119(24):e592-3. https://doi.org/10.1161/CIRCULATIONAHA.109.852053

9. Moffa PJ, Sanchez PCR. Eletrocardiograma normal e patológico. São Paulo: Roca; 2001.

10. Marriott HJ. Differential diagnosis of supraventricular and ventricular tachycardia. Cardiology. 1990;77(3):209-20. https://doi. org/10.1159/000174602

11. Vereckei A, Duray G, Szénási G, Altemose GT, Miller JM. New algorithm using only lead aVR for differential diagnosis of wide QRS complex tachycardia. Heart Rhythm. 2008;5(1):89-98. https://doi.org/10.1016/j.hrthm.2007.09.020

12. Mann D, Zipes D, Libby P, Bonow R. Braunwald's Heart Disease: A Textbook of Cardiovascular Medicine, 10 th ed, Saunders Elsevier, 2014.

13. Wellens HJ, Bär FW, Lie KI. The value of the electrocardiogram in the differential diagnosis of a tachycardia with a widened QRS complex. Am J Med 1978;64(1):27-33. https://doi.org/10.1016/002-9343(78)90176-6

14. Pava LF, Perafán P, Badiel M, Arango JJ, Mont L, Morillo CA, et al. R-wave peak time at DIl: a new criterion for differentiating between wide complex QRS tachycardias. Heart Rhythm. 2010;7(7):922-6. https://doi.org/10.1016/j.hrthm.2010.03.001

15. Take Y, Morita H. Fragmented QRS: What Is The Meaning? Indian Pacing Electrophysiol J. 2012;12(5):213-25. https://doi.org/10.1016/ s0972-6292(16)30544-7

16. Kindwall KE, Brown J, Josephson ME. Electrocardiographic criteria for ventricular tachycardia in wide complex left bundle branch block morphology tachycardias. Am J Cardiol. 1988;61(15):1279-83. https://doi.org/10.1016/0002-9149(88)91169-1

17. Santos JAB, Galvão Filho SS, Vasconcelos JTM, Pinna Junior BJB, Vitorino EMS, Martienzo ALA, et al. Diagnóstico de taquicardia ventricular do tipo fascicular através da cardioexploração eletrofisiológica transesofágica (Cete). Reblampa. 1998;11(1):31:9.

18. Lerman BB, Stein KM, Markovitz SM. Mechanism of idiopathic left ventricular tachycardia. J Cardiovasc Electrophysiol. 1997;8(5):57183. https://doi.org/10.1111/j.1540-8167.1997.tb00826.x 
19. Tchou P, Mehdirad AA. Bundle branch reentry ventricular tachycardia. Pacing Clin Electrophysiol. 1995; 18(7):1427-37. https://doi. org/10.1111/j.1540-8159.1995.tb02605.x

20. Zipes DP, Jalife J. Cardiac Electrophysiology from Cell to Bedside. Philadelphia: Saunders/Elsevier; 2009. Atrioventricular reentry and variants.

21. Steurer G, Gürsoy S, Frey B, Simonis F, Andries E, Kuck K, et al. The differential diagnosis on the electrocardiogram between ventricular tachycardia and preexcited tachycardia. Clin Cardiol. 1994;17(6):306-8. https://doi.org/10.1002/clc.4960170606 